\title{
La tecnología 5G: ¿Determinante en la disputa por la hegemonía económica global entre China y Estados Unidos en el siglo XXI?
}

5G technology: Is it a determinant in the dispute for global economic hegemony between China and the United States in the 21 st century?

>> Mtra. Tania Anahí Díaz González Universidad de Colima (México) tania_diaz@ucol.mx

\section{Dr. Juan González García}

Profesor investigador, Universidad de Colima (México)

jgogar@ucol.mx

Dr. Ángel Licona Michel

Profesor investigador, Universidad de

Colima (México)

almichel@ucol.mx

Recibido: 26 de septiembre Aprobado: 9 de noviembre Publicado: 17 de diciembre

\section{Resumen}

La tecnología de quinta generación (5G) es actualmente la última manifestación del avance científico tecnológico en el mundo, la cual está impactando en la esfera económica, política, en las relaciones internacionales y en la seguridad nacional e internacional de los países. Hasta ahora, China ha liderado su implementación, lo que la ha hecho adquirir un papel relevante en la gobernanza mundial, de tal modo que ha dado lugar a un enfrentamiento con Estados Unidos en el contexto de la disputa por la hegemonía económica global.

La hipótesis del texto es que el avance que tiene China en la tecnología $5 \mathrm{G}$ será un catalizador para su posicionamiento como primera potencia económica global. En el artículo se analizan las características de la tecnología 5G; posteriormente su importancia en China y el mundo; asimismo, se revisa la carrera hacia la supremacía tecnológica, reconociendo el papel de la 5G en 
los sectores claves del posicionamiento económico de China en la economía global, lo que a la postre, le facilitará su ascenso y predominio en la lucha directa que mantiene con Estados Unidos.

\section{Palabras claves}

5G, hegemonía, economía, tecnología, guerra comercial

\section{Abstract}

The fifth generation $(5 \mathrm{G})$ technology is currently the latest manifestation of scientific and technological development in the world, which is impacting the economic, political, and international relations and the national and international security of countries. So far, China has led its implementation, which has lead it to acquire a relevant role in world governance to such an extent that it has given rise to a confrontation with the United States in the context of the dispute for global economic hegemony.

The hypothesis of the text is that the progress made by China in $5 \mathrm{G}$ technology will be a catalyst for it to be positioned as the first global economic power. The article analyzes the characteristics of $5 \mathrm{G}$ technology and its importance in China and the world; it also reviews the race toward technological supremacy, recognizing the role of $5 \mathrm{G}$ in the key sectors of China's economic positioning in the global economy, which will ultimately enable its rise and predominance in the direct battle with the United States.

\section{Keywords}

$5 G$, hegemony, economy, technology, trade war

\section{Introducción}

El mundo en el siglo XXI se presenta como un gran sistema complejo, en el que las naciones, las empresas y la población están experimentando múltiples cambios. Si bien la constante en la sociedad y economía internacional es el cambio, este se presenta a diversos ritmos, unas veces de manera gradual e incremental y, otras, revolucionario y acelerado. Con la globalización económica, los cambios que se han presentado en el mundo van delineando el perfil del siglo XXI: la sociedad y la economía global del cambio acelerado.

Así, el rápido desarrollo que ha tenido la tecnología ha contribuido a este cambio acelerado, lo que, particularmente en las últimas décadas, impacta directamente en la configuración de las relaciones entre naciones. Se considera que aquella nación que tenga ventajas en el desarrollo tecnológico gozará de sus beneficios directamente en su crecimiento económico, estabilidad y presencia en el sistema internacional. Este es el caso de China, país que ha fortalecido sus capacidades tecnológicas, generándole una ventaja en los sectores que están dominando el mercado mundial, por lo que su papel en el contexto internacional está fortaleciéndose cada vez más y compitiendo con Estados Unidos.

El nuevo orden económico internacional (NOEI), que surgió después de la Segunda Guerra Mundial (SGM), con Estados Unidos como líder en Occidente, tuvo décadas de estabilidad y certidumbre; sin embargo, desde finales de la década de los setenta del siglo XX, y más fortalecido en el siglo XXI, China experimentó una reemergencia económica y política en la esfera internacional, que se ha caracterizado por una tasa de crecimiento económico alta y sostenida. Con esto, viene disputándole ese liderazgo económico a Estados Unidos, haciendo de la carrera tecnológica entre ambos países un claro ejemplo de competencia. 
Luego del experimento del socialismo real de 1949-1978, China dio un giro a su modelo de desarrollo económico; hizo reforma económica y apertura externa, se incorporó al mecanismo de mercado y, en general, cambió las reglas del juego económico para los agentes económicos internos y del exterior. Así, el gigante asiático se presenta en la actualidad como uno de los países que más impulsa la transformación económica por medio de la incorporación de las tecnologías de información y comunicación (TIC) y de un decidido apoyo a la educación, desarrollo tecnológico, ciencia, tecnología e innovación. Estos aspectos, en conjunto, pretenden convertir a China en una de las economías más importantes del mundo a mediados del presente siglo XXI (Cornejo y González, 2009).

Por lo tanto, para comprender el actual posicionamiento de China como líder económico y tecnológico, es importante conocer las áreas particulares en las que se ha enfocado, como la llamada tecnología 5G. En este contexto, la $5 \mathrm{G}$ se presenta como el principal objetivo de estados y empresas para llegar a la supremacía del sistema de reproducción económico, político y social imperante, sea capitalista puro o con características chinas.

El objetivo del artículo es analizar la importancia de la 5G en China, así como el impacto que esta ha tenido en distintos sectores. Se plantea la hipótesis de que el desarrollo de la 5G que ha registrado China en la actualidad, está permitiéndole inclinar la lucha por la supremacía económica global que sostiene con Estados Unidos. Es decir, se considera que el avance que tiene China en la tecnología $5 \mathrm{G}$ será un catalizador para su posicionamiento como primera potencia económica global en el presente siglo.

El artículo se estructura de la siguiente manera: en la primera sección, se define y caracteriza la 5G; en la segunda, se desarrolla su importancia, particularmente ejemplificando las aplicaciones en la sociedad; en la tercera sección se describe el conflicto entre China y Estados Unidos; finalmente, previo a las conclusiones se analiza el papel de la 5G como factor prima facie en la disputa por la hegemonía económica global y los sectores clave involucrados.

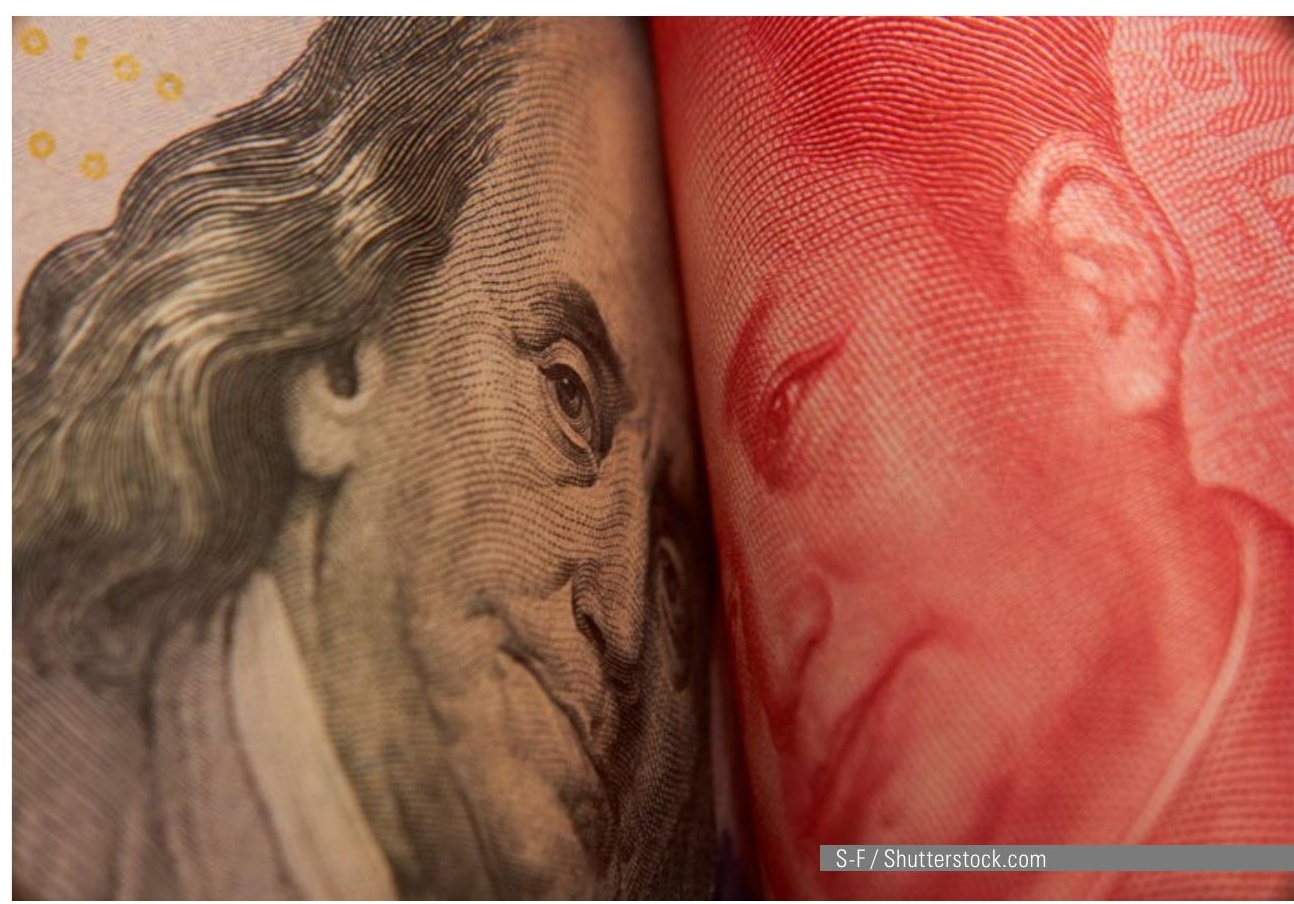




\section{Definición y características de la tecnología 5G}

A finales del siglo XX, las redes de comunicación derivadas del avance tecnológico permitieron que la sociedad tuviera a su disposición mayores herramientas de comunicación con el uso de la tecnología móvil, de esta forma, a partir de las tecnologías de segunda, tercera y cuarta generación, se han dado avances paulatinamente más extraordinarios en cada actualización, pasando de las llamadas móviles, a los mensajes de texto y a la navegación en internet. ${ }^{1}$

Además de mejorar y brindar mayores servicios, la 5G se plantea como objetivo una mayor velocidad en la transmisión de datos. También posibilita un ecosistema capaz de entregar eficientemente servicios inalámbricos entre máquinas, cosas y personas, revolucionando en todo sentido la forma de comunicarnos mediante conexiones fiables, eficientes y eficaces (Batista y Díaz, 2019).

Es importante considerar que la 5G proveerá Comunicaciones Ultra Confiables de Baja Latencia (URLLC), esto significa que hará posible admitir casos de uso que requieren confiabilidad muy alta, latencia extremadamente baja y eficiencia energética; como ejemplos de estos casos se incluyen la automatización industrial, la conducción autónoma, la cirugía remota, el soporte a los servicios de entretenimiento, entre otros (Kahuha, 2021).

En este contexto, la transmisión de múltiples antenas ya desempeña un papel importante para las generaciones actuales de comunicaciones móviles y jugará un rol aún más importante en la era de la 5G, la trasmisión de múltiples antenas o nodos servirán como base sólida con el objetivo de aumentar el volumen de los datos 1.000 veces en comparación con los actuales, así como el número de dispositivos conectados en las redes (González y Salamanca, 2016).

Hace aproximadamente una década se planteó que la 5G utilizará las tecnologías inalámbricas que conocemos hoy, pero en un solo sistema integral, brindando así comunicación masiva desde cualquier dispositivo, con una latencia sumamente baja, imperceptible para el ser humano, aún en comunicaciones de largas distancias, con una velocidad de transmisión 10 veces mayor a la de 4G (Tamayo, 2017).

La tecnología $5 G$ tiene consigo ventajas considerables en comparación con tecnologías anteriores, en específico desde la 4G, con un ancho de banda de datos de 1 Giga byte por segundo (Gbps) o superior y que permite la bidireccionalidad. Se propone que sea globalmente accesible, que permita la comunicación directa entre dispositivos, acepte una mayor densidad de agrupamiento de usuarios y traiga consigo una mayor eficiencia energética (González y Salamanca, 2016).

Se señala que la tecnología 5G tiene baja capacidad de atravesar obstáculos, por eso requiere un mayor número de transmisores para rodearlos; además, algunos dispositivos no serán compatibles con la red 5G, por lo que requerirán cambios; también se destaca que el costo de la infraestructura para desplegar la 5G es elevado (Batista y Díaz, 2019). No obstante, los países han apostado por continuar desarrollando las redes 5G, si bien estas todavía no se encuentran totalmente implementadas en el mundo, e incluso, ya existen planes para que antes de finalizar la tercera década del siglo XXI estén en funcionamiento las redes de sexta generación.

Esta tecnología está comenzando a generar grandes cambios en la sociedad, pero no solo como usuarios y para comunicarse de manera indi-

1 La red de comunicación inalámbrica de primera generación (1G) fue introducida en 1980; en 1990 se introdujo la de segunda generación $(2 \mathrm{G})$ con señales de radio digitales, ofreciendo mayor seguridad y el servicio de mensajes de texto. La tercera generación (3G) surgió a principios del 2000, misma que permitió el acceso a internet y en 2010 apareció la cuarta generación (4G) que alcanzaba velocidades de $10 \mathrm{Mbps}$, lo que permite reproducciones en alta resolución (Patel, et al, 2018). 
vidual, el enfoque va más allá: tiene que ver con la economía. Una de las motivaciones que impulsan a los países y empresas a seguir en la creación de infraestructura para contar con esta tecnología, es su impacto económico.

En 2016 las tecnologías y servicios móviles generaron el 4,4\% del PIB en el mundo (3,3 billones de dólares) e incrementaron al 4,7\% (4,1 billones de dólares) en 2019. Muy probablemente en 2020 su participación en el PIB mundial represente el 4,9\% (4,2 billones de dólares) y se prevé que, a finales de 2024, supere ese 4,9\% y la cifra alcance los 4,9 billones de dólares (Corral, 2020: 24-25).

En definitiva, la tecnología 5G ha llegado para revolucionar las comunicaciones, facilitar el transporte, innovar en el área médica e incrementar la productividad. Considerando este último aspecto, es importante conocer que el impacto económico de la tecnología móvil 5G se mide a través de su capacidad para aumentar la eficiencia y la productividad, lo cual se comprueba con las estimaciones de que con la $5 \mathrm{G}$ y sus aplicaciones se impulsará el PIB mundial en 1,3 billones de dólares para 2030 (PWC, 2021).

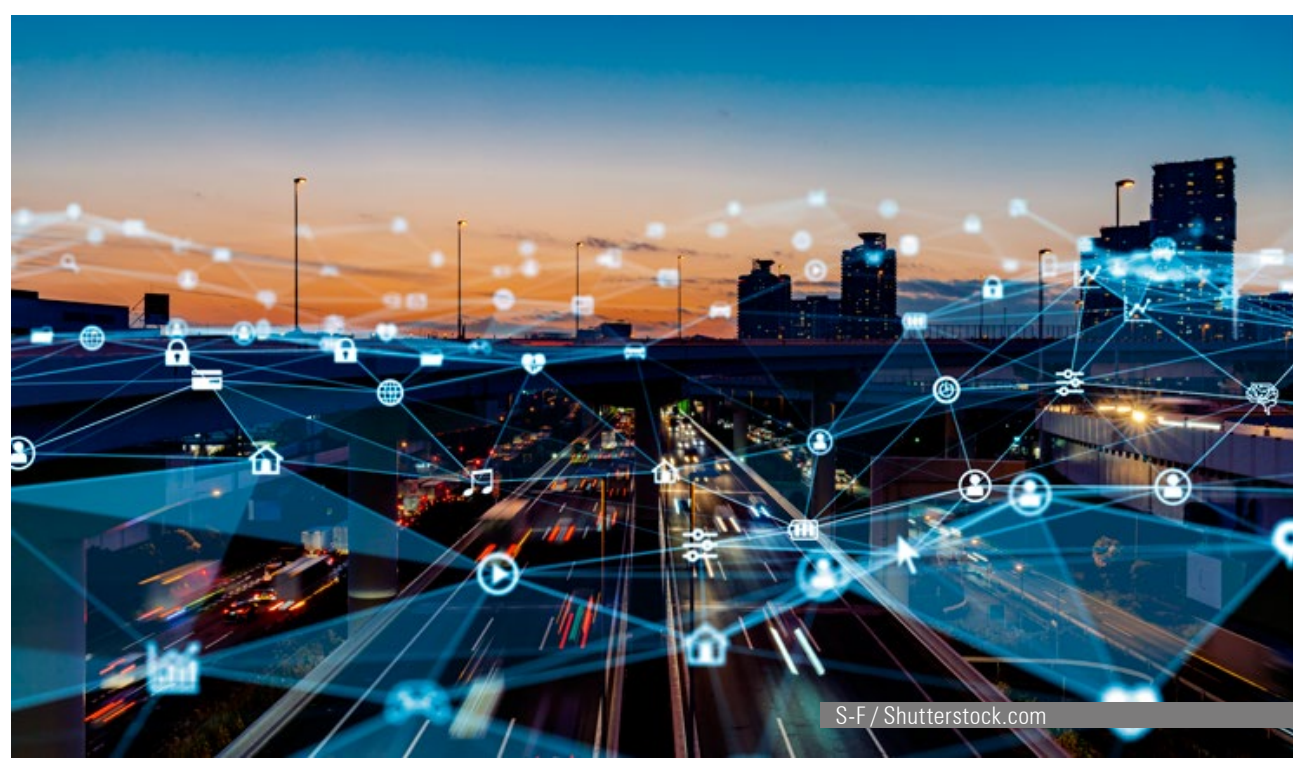

\section{La importancia de la tecnología 5G en China y el mundo: apli- caciones en la sociedad}

China ha destacado por su rápido crecimiento y posicionamiento económico a partir del proceso de apertura y modernización en 1978. Entre los años 1978 a 2020 su promedio de crecimiento económico fue del 9,14\% anual (Banco Mundial, 2021). En los últimos años el país ha tenido un avance tecnológico sumamente significativo, "el gasto anual en investigación y desarrollo $(I+D)$ de China creció 169 veces, de alrededor de 14.300 millones de yuanes (2.210 millones de dólares) a principios de la década de 1990 a 2,44 billones de yuanes en 2020" (Global Times, 2021).

Dicha inversión se refleja tanto en el sector productivo, como en la vida diaria de los ciudadanos, pues el avance tecnológico en general ha tenido un impacto directo en el mejoramiento de la industria, los servicios y los productos. El mundo ha progresado en el avance y aplicación de las tecnologías, asunto muy notorio en China, donde el desarrollo tecnológico se refleja en la utilización de los pagos digitales, el comercio electrónico, las redes móviles, el mejoramiento de los transportes gracias a los trenes de alta velocidad, etc. (Global Times, 2021). 
De hecho, gracias al avance que China registra en la red 5G, al marco normativo y a la seguridad de los sistemas de pago, las transacciones realizadas por los consumidores, ya sea familias, empresas o el mismo gobierno, se han incrementado en las modalidades B2B (business to business); B2C (business to consumer) y B2G (business to goverment) logrando llevar a China a convertirse en uno de los países con mayor crecimiento y dinamismo del comercio electrónico o e-commerce en el mundo (González, 2020).

La $5 G$ ha llegado para mejorar este tipo de experiencias y seguir impactando en la vida diaria de las personas, no solo en China, sino en el mundo. Con la $5 \mathrm{G}$ es posible mantener conectividad en lugares de forma masiva y sin que se presenten problemas por un mayor número de usuarios en la red; este es un aspecto fundamental considerando que en China en 2020 se alcanzaron los 998,9 millones de usuarios de internet (Statista, 2021), lo cual refleja que el $70 \%$ de la población en China tiene tanto acceso a la red, como a dispositivos electrónicos.

A continuación, se mencionarán únicamente tres sectores en los que la tecnología $5 \mathrm{G}$ ha generado un impacto en la sociedad china: el comercio electrónico (e-commerce), la educación y la salud, todos ellos generan una influencia directa, tanto en la vida diaria, como en la economía.

Comprar y vender tanto productos como servicios a través de las redes digitales se ha convertido en una tendencia en China, misma que ha adquirido bastante popularidad en otros países asiáticos y occidentales, fenómeno que también se ha intensificado a raíz de la pandemia ocasionada por la covid-19. Alibaba, la popular corporación china de comercio electrónico, ha sido testigo de la evolución industrial desde las primeras etapas del e-commerce; actualmente posee aproximadamente el $50 \%$ del negocio de plataformas de comercio electrónico y su participación aumenta al $90 \%$ del negocio si se incluye el comercio móvil (Kwak, et al., 2019).

El comercio electrónico tiene bastantes beneficios, crea competencia y mayores oportunidades para el consumidor, abriéndose el número de posibilidades al poder elegir entre la oferta de distintos vendedores. El e-commerce en China ha mejorado y también se ha convertido en un motor que impulsa el desarrollo económico del país (State Council, 2020).

A manera de ejemplo, el grupo Alibaba registró en 2020 ingresos por 473.680 millones de yuanes en ventas online en China (72,3 mil millones de dólares estadounidenses). Con esta cifra, se constata la importancia del mercado chino, debido al número de consumidores, con una población de 1.439,3 millones de habitantes (Fondo de Población de las Naciones Unidas [UNFPA], 2021), de los cuáles $70 \%$ tiene acceso a internet y favorece las posibilidades de crecimiento en el comercio en línea para satisfacer cualquier necesidad.

Específicamente, la escala y la calidad de los bienes y servicios se han acelerado en estos últimos años, en los que la escala del mercado de comercio electrónico de China continúa liderando el mundo, y las capacidades de servicio y los niveles de aplicación han mejorado aún más, reafirmando la importancia que este nuevo tipo de consumo representa en la era moderna de este país.

Por otro lado, el número de usuarios de internet en China está cercano a los 1.000 millones, lo que explica que el volumen de transacciones de comercio electrónico nacional ha alcanzado los 34,81 billones de renminbi (RMB), de los cuales las ventas minoristas en línea han alcanzado los 10,63 billones de RMB, esto refleja un aumento anual del 16,5\%. Además, según el informe anual de 2019, los empleados de comercio electrónico alcanzaron el número de 51'256.500 millones (Ministry of Commerce, 2020).

El gobierno ha concedido importancia al desarrollo del comercio electrónico, si bien las ventas del comercio se centran en las provincias de Guang- 
dong, con el 23,76\%; de Zhejiang, con el 16,54 \%; y en la ciudad de Shanghái, con el 11,63\%. Por su parte, en las regiones rurales el comercio electrónico ha incrementado su participación, alcanzado en 2019 1,7 billones de RMB en ventas, lo que significa un crecimiento del 19,1 \% respecto al año 2018 (Ministry of Commerce, 2020). Con estas cifras, además del significativo aumento, se observa beneficio al mayor número de habitantes, incluyendo las zonas rurales, y un incremento en la creación de empleos.

En la actualidad, la $5 \mathrm{G}$ y el comercio electrónico tienen una fuerte relación, es una realidad en la que la interconectividad, la posibilidad de acceder a internet desde prácticamente cualquier lugar y tener una experiencia de compra cada vez mejor, es sumamente común en la sociedad del siglo XXI y particularmente en China.

Una mayor accesibilidad para los usuarios, así como la velocidad más rápida de las redes $5 \mathrm{G}$ y las pantallas de alta resolución de los dispositivos habilitados para 5G, pueden llevar a un mayor grado de disfrute del cliente al participar en actividades de comercio electrónico, a dedicar más tiempo a navegar en sitios web de comercio electrónico y ha realizar más compras en línea (Kshetri, 2018).

Se estima que en 2020 las ventas del comercio electrónico minorista en todo el mundo registraron una tasa de crecimiento del $27,6 \%$, con ventas que superaron los 4.280 trillones de dólares. Se considera que para el año 2021 el crecimiento mundial del e-commerce será del 14,3 \%, con ventas de 4.891 trillones de dólares (Crammer-Flood, 2021).

Otro de los sectores que ha tenido un desarrollo más rápido a partir de la penetración de la 5G es la educación. La informatización entendida como el uso de medios informáticos para el desarrollo de diversas actividades es considerada por Lu y Sun (2020) como el poder central de la modernización de la educación, la aplicación de la 5G para la enseñanza e investigación puede brindar una educación con mayores alcances, utilizando sistemas interactivos de enseñanza a larga distancia y en tiempo real.

De manera general, la 5G permite potenciar distintas áreas de la educación y la forma tradicional del proceso de enseñanza aprendizaje en el mundo, entre ellas, la gestión de aulas inteligentes para un mayor control y seguimiento, la descarga de videos en segundos, un aprendizaje más flexible independientemente de la distancia o la ubicación y mayor asistencia para estudiantes con necesidades especiales. La 5G permitirá a los robots ser asistentes de tiempo completo y apoyar a los maestros respondiendo de inmediato para ayudar con los ejercicios de aprendizaje, mayor difusión de aplicaciones que exigen baja latencia y procesamiento de información más rápido, por lo cual se desarrollarán aplicaciones en un mayor número de áreas de conocimiento, y el internet táctil de baja latencia facilitará el aprendizaje y la educación a distancia mediante la superposición táctil de profesores y estudiantes (Mokhtar y Ahmad, 2020).

Finalmente, otro de los sectores que se ha beneficiado del desarrollo tecnológico y de la $5 \mathrm{G}$ es el de la salud, pues esta proporciona un soporte de comunicación crucial en los campos de comando de emergencia, el transporte y la gestión hospitalaria. También desempeña un papel en la consulta a distancia, la aplicación de robots médicos y el uso de termómetros infrarrojos (Ouyang, 2020).

Existen enormes oportunidades a través del uso de la $5 \mathrm{G}$ en una variedad de sectores para conectar el mundo de la atención médica de manera creativa, el uso de dispositivos móviles, así como de sensores y equipos de monitoreo remoto está aumentando y habrá un incremento en pacientes que reciben imágenes, diagnóstico o tratamiento a través de tecnología digital (West, 2016).

La aplicación y beneficio de la $5 \mathrm{G}$ en la vida cotidiana se observó particularmente durante la expansión de la pandemia de la covid-19. En este 
sentido, el uso de la tecnología también fue una herramienta para el gobierno en el combate a la enfermedad. La aplicación de las tecnologías soportadas en $5 G$ tuvieron diversas aplicaciones para el monitoreo de los casos o las consultas por video, para diagnosticar a los enfermos, así como el uso de termómetros infrarrojo.

Considerando que la fiebre es uno de los principales síntomas de la covid-19, en China, como en otros países, se utilizan sistemas basados en inteligencia artificial en cruces de transporte, edificios de oficinas y comunidades para identificar las altas temperaturas corporales de un flujo de personas en movimiento, basado en la forma del cuerpo y otra información facial, el sistema inteligente ayuda al personal a identificar y monitorear rápidamente a las personas con temperatura corporal anormal. Además, de conformidad con las regulaciones, las ciudades chinas están utilizando plataformas de análisis de big data para rastrear contactos cercanos de pacientes o casos sospechosos (Soldani, 2020).

Como resultado de las características y ventajas de la $5 \mathrm{G}$ mencionadas anteriormente, tales como la conexión de alta velocidad, alta confiabilidad y baja latencia, el sistema de atención médica se ha beneficiado de tiempos de respuesta mejorados, monitoreo de pacientes, recopilación y análisis de datos, colaboración remota y asignación de recursos. También establece un ejemplo para las principales plataformas de respuesta a emergencias públicas innovadoras, digitalizadas y basadas en la nube (DTTL, 2020).

Estas estrategias pueden reflejarse en un menor número de personal empleado para atender y dar seguimiento a los casos, así como en los controles de empresas, centros comerciales e instituciones, lo que al final ofrece como resultado la disminución de casos o personas expuestas al virus.

En este contexto, se debe de considerar que la covid-19 ha sido un catalizador para comercializar el uso de la 5G en China, y aún falta bastante para expandir su uso en todo el país. En efecto, en septiembre de 2020 Beijing se convirtió en la segunda ciudad, después de Shenzhen, en tener una cobertura total de la tecnología 5G (Tomás, 2020). El avance que está teniendo este país tiene un gran significado a nivel internacional y la cobertura aumentará rápidamente, se prevé que las conexiones $5 \mathrm{G}$ chinas escalarán rápidamente con el tiempo, para llegar a 428 millones de habitantes en el territorio de ese país en 2025 (CAICT, 2017).

Como se puede constatar, los tres sectores mencionados son ejemplos de los distintos beneficios que tiene la aplicación de la 5G en la economía y la vida diaria en China, puesto que sus aplicaciones, que paulatinamente están difundiendose más en las sociedades alrededor del mundo, brindan múltiples beneficios. Es decir, la 5G incrementa la productividad de sectores como la manufactura, la agricultura, las finanzas y los sistemas de distribución; también traerá mayores avances en la salud, electrónica, educación, transporte público y privado.

Además, se espera que contribuya a la mejora de las medidas de seguridad pública, como las respuestas de seguridad y desastres en tiempo real, así como a resolver la brecha médica y educativa entre las regiones. La expectativa es que, con la 5G se acelere la cuarta revolución industrial, en la que todos los objetos están conectados (García, et al., 2020). 


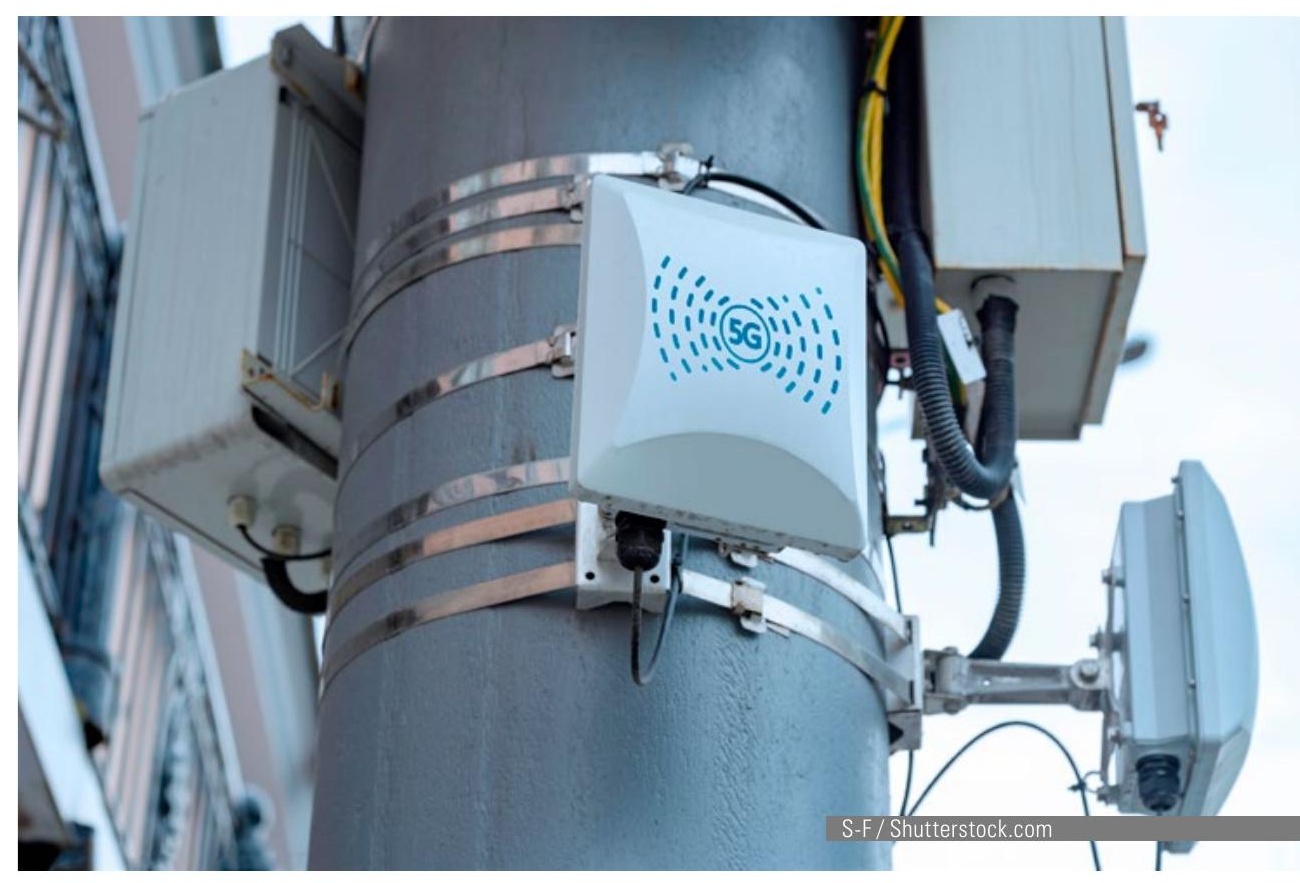

\section{El conflicto entre China y Estados Unidos: hacia la supremacía tecnológica}

Existen diversos problemas y cambios que están definiendo la realidad y las tendencias hacia la primera mitad del siglo XXI. Oropeza (2019) afirma que la problemática de nuestro tiempo podría estructurarse en dos claras etapas que están poniendo a prueba la capacidad de la sociedad global para resolverlas. La primera de ellas se caracteriza por el choque y el desenlace de las dos tendencias: el ascendente predominio por parte de China respecto a Estados Unidos (la inevitabilidad China) y su consecuencia directa, el traslado de la era del Atlántico al Pacífico (inevitabilidad asiática). Por ende, en la actualidad el debate del dominio entre China y Estados Unidos se posiciona como una problemática actual y presente en nuestros días.

Tradicionalmente, en el campo de las relaciones internacionales el poder de cada Estado dependía principalmente de sus recursos económicos y militares, derivando en un orden determinado por el dominio de estos recursos. Sin embargo, en la actualidad la supremacía geopolítica y económica se determinará por aquellos poderes que manejen la Inteligencia Artificial (IA) y la 5G. De esta forma Rusia y China están desafiando el orden mundial liderado por Estados Unidos por una nueva competencia tecnológica en economía, modernización militar y medios de control social que capitalizan los avances que proporcionan estas tecnologías (Fricke, 2020).

Desde el punto de vista de China, su principal fortaleza de la estrategia para seguir desarrollando la $5 \mathrm{G}$ es la existencia de un sistema centralizado en el que el gobierno interviene directamente en la economía con el fin de perseguir la eficiencia de la producción, con acciones como blindar las empresas nacionales de la competencia extranjera, así como asignar fondos impulsados por las intenciones políticas. En este sentido, la planificación proporciona a China una ventaja comparativa en la carrera $5 \mathrm{G}$ en relación con Estados Unidos y la Unión Europea, garantizando una coordinación entre las empresas de telecomunicaciones, lo que facilita la creación de sinergias 
en investigación y desarrollo $(I+D)$ e incluso implica financiación pública en apoyo de la innovación (Mariani y Bertolini, 2019).

Naturalmente, la centralización pública de la estrategia $5 \mathrm{G}$ es criticada e incluso vista como una serie de prácticas desleales frente a la competitividad internacional, ya que, si bien para China la forma en la que dirige su economía es vista como algo natural para fortalecer a sus empresas, desde la perspectiva de la competencia internacional, se ve como una debilidad para la competencia extranjera, que no recibe fondos directos de sus países.

Efectivamente, el sistema dirigista chino cuenta con el potencial de proporcionar beneficios para el desarrollo económico futuro de la 5G. Esto significa que puede inyectar miles de millones de dólares al sector $5 G$ en los próximos años, preparando el escenario para nuevos avances en productividad e innovación. La conectividad sin precedentes que proporcionará la 5G es fundamental para aprovechar todo el potencial del internet de las cosas y la inteligencia artificial, habilitando nuevas industrias y contribuyendo a una economía digital dinámica; además, dada la importancia de la 5G para la competitividad nacional, esta se ha caracterizado como eje fundamental de la carrera entre China y Estados Unidos (Kania, 2019).

Por otra parte, desde el punto de vista geopolítico, que exista una mejoría en la conectividad y la velocidad tiene efectos directos en la tecnología empleada en el campo militar, brindando ventajas al país que posea los mejores avances. Por ello se ha dado tanta importancia al avance en el desarrollo de la 5G de China, de hecho, el gobierno estadounidense enmarca el tema de la 5G como una cuestión de seguridad nacional, apoyando su argumento en tres pilares: seguridad, economía y enfrentamiento sistémico (Rühlig, et. al, 2019).

Más allá del argumento basado en los tres pilares mencionados, la carrera por la $5 \mathrm{G}$, además de los efectos comerciales que ha tenido, puede ser considerada como una carrera armamentista. Es decir, en términos de seguridad, el temor es que los equipos de infraestructura de Huawei puedan facilitar el espionaje político o industrial. Económicamente, busca proteger a las empresas estadounidenses de una posible competencia desleal y evitar la dependencia excesiva de la economía y la tecnología china.

Este es el contexto que debe tenerse en mente al analizar el argumento mencionado anteriormente sobre el Estado dirigista chino y el posible apalancamiento político del que se ha visto beneficiado Huawei. El último argumento es la preocupación causada porque la tecnología de red de acceso por radio, que es esencial para el despliegue de la $5 \mathrm{G}$, la suministran actualmente solo tres proveedores en el mundo: Huawei, Ericsson y Nokia (Rühlig, et al., 2019).

Como resultado del debate en torno a la competencia desleal y a la actuación de los gobiernos, desde 2018 el expresidente Donald Trump lanzó un ataque de múltiples frentes contra la empresa china Huawei. En el frente legal, demandó a la firma y buscó extraditar a un alto ejecutivo para enfrentar cargos criminales; en el comercial, ha tomado medidas para prohibir los equipos de Huawei en las redes de telecomunicaciones nacionales y ha hecho campaña para que sus aliados y otros sigan su ejemplo (Kennedy, 2020).

En medios de comunicación, a través de declaraciones e incluso utilizando las redes sociales se ha visto la preocupación del gobierno estadounidense respecto al avance de Huawei y su estrategia para aislarla, no solo en el ámbito interno, sino respecto a sus aliados en Europa. La importancia de Huawei en la industria de las tecnologías de la información y comunicación es cada vez más notoria. En efecto, la participación en el mercado global de Huawei para equipos de proveedores de servicios es, con cifras de 2019, mayor que la de Ericsson y Nokia juntas. A partir de 2016 Huawei suministró más de la mitad de las 537 redes móviles $4 \mathrm{G}$ a del mundo y 59 de las 90 redes 4,5G, los proveedores europeos utilizan equipos Nokia y Ericsson, pero cada vez más recurren a Huawei por tener mejores precios y tecnología (Donahue, 2019). 
En mayo de 2019, el Departamento de Estado de Estados Unidos incluyó a Huawei en la lista negra del mercado estadounidense, argumentando competencia desleal y robo de propiedad intelectual, decisión respaldada por otras empresas tecnológicas ubicadas en ese país; Google restringió el alcance de los teléfonos inteligentes de Huawei a sus aplicaciones; Intel, Broadcom y Qualcomm cortaron sus lazos con Huawei, e incluso se impusieron restricciones a empresas estadounidenses que hicieran acuerdos comerciales con determinadas empresas chinas (Tekir, 2020).

En respuesta a las decisiones de Estados Unidos, Huawei publicó un documento que responde a las acusaciones planteadas por el Departamento de Estado, negando estar subsidiada por el gobierno chino o involucrada en el robo intelectual. Asegurando que respeta la seguridad incluso más que Estados Unidos; además, en la respuesta advirtió a los países sobre los crecientes costos y posibles retrasos en el desarrollo de la 5G, en caso de que la empresa sea prohibida (Tekir, 2020).

Por su parte, el gobierno de China respondió a las acciones y medidas del gobierno estadounidense aplicando aranceles del $5 \%$ al $25 \%$ a productos de este país. El 15 de enero de 2020, considerando las afectaciones ocasionadas en 2019 tanto en términos de comercio como de inversiones por las acciones de ambas naciones, se firmó una tregua entre los gobiernos. Sin embargo, previamente, el impacto de los aranceles totales estadounidenses aplicados exclusivamente a productos chinos alcanzaron los 550 billones de dólares y los aranceles chinos aplicados exclusivamente a productos estadounidenses los 185 billones de dólares (Wong y Chipman, 2020).

La pandemia de la covid-19, en tanto factor exógeno al conflicto comercial y tecnológico entre ambos países, propició que priorizaran la resolución de otros problemas y, de alguna manera, se generó una tregua de varios meses en 2020. Sin embargo, lejos de solucionarse el conflicto, este entró en un impasse, hasta que el, en ese momento, presidente electo Joseph Biden, estableció que no eliminaría los aranceles impuestos a China de inmediato (Zhen, 2020).

La decisión se justifica porque el avance tecnológico chino ha sido objeto de desconfianza; en este contexto, se tiene que considerar que los alcances de las medidas no han generado pérdidas económicas únicamente para esos dos países, sino que, contemplando que vivimos en un sistema interdependiente, los resultados han impactado directamente en otros países.

Entre las recientes respuestas del gobierno chino frente a esta guerra comercial se encuentran la Ley de Control de Exportaciones, una legislación que restringe la exportación de artículos controlados, como artículos nucleares, militares y artículos de "doble uso" que pueden utilizarse tanto para fines civiles como militares, así como otros elementos que se consideran vitales para la protección de la seguridad nacional de China. Con ello, se asume como una posible herramienta para restringir la venta de tecnología sensible en el mundo (BBC, 2020). Pese a las afectaciones ya mencionadas y la tregua existente, una vez comenzado el enfrentamiento y la carrera hacia el predominio global, la tendencia se mantendrá con este tipo de legislaciones y acciones.

La confrontación entre Estados Unidos y China en asuntos referentes a las TIC se considera una "guerra fría tecnológica" en la que, el temor de los críticos de Huawei no es la inseguridad de la red, sino la dependencia tecnológica. La interdependencia se ha identificado en general como una fuerza estabilizadora, sobre todo porque eleva los costos de la coerción y en el extremo de la guerra (Rühlig y Björk, 2020). Al existir un equilibrio de poder, las posibilidades de los enfrentamientos se disminuyen y por ende existe una mayor armonía en el sistema internacional.

Considerando la breve descripción de los enfrentamientos suscitados a partir de 2018, se asume que definitivamente nos encontramos en medio de 
una fuerte competencia mundial, donde la carrera por tomar el liderazgo en tecnologías avanzadas como la Inteligencia Artificial, la computación cuántica y las redes $5 \mathrm{G}$ determinarán el futuro equilibrio del poder geopolítico. Y si bien, Estados Unidos y sus socios alguna vez disfrutaron de una clara ventaja, esa ventaja ya no puede asumirse, pues China avanza a toda velocidad y reclama un liderazgo tecnológico (Rogers y Nye, 2019).

Sea cual sea la estrategia seguida por ambas naciones, es posible considerar que "ganar la carrera 5G representaría no solo un éxito económico, sino también un logro político fundamental" (Mariani y Bertolini, 2019: 7). Estados Unidos ha sido un actor elemental en el establecimiento del orden económico internacional de postguerra y dentro de las instituciones surgidas a partir de la SGM, ha tenido ventajas económicas, políticas, militares y tecnológicas, mismas que no son suficientes para ostentar su lugar frente a una China que ha respondido a los desafíos internacionales con una estrategia centrada en sus necesidades y que en pocos años se ha propuesto afianzar un desarrollo potenciado por la innovación y el avance tecnológico.

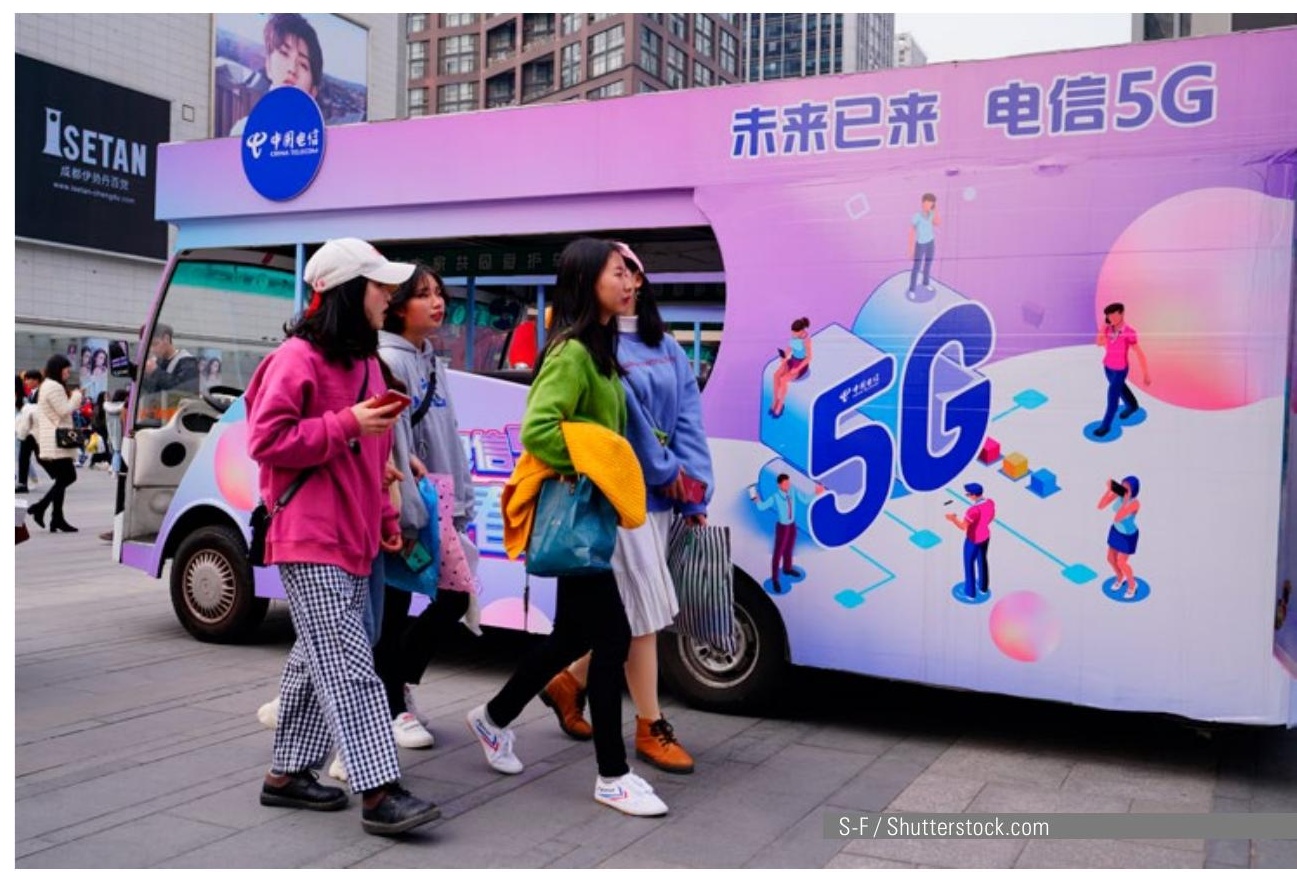

\section{Papel de la $5 G$ en los sectores claves del posicionamiento eco- nómico de China en la economía global}

China definitivamente se ha propuesto desempeñar un papel más activo y estratégico en el sistema internacional, para ello ha sido necesario modificar su estrategia de crecimiento y aumentar sus lazos de cooperación internacional. Desde su apertura, ha buscado incrementar su nivel de industrialización y posicionarse a nivel internacional y, con la llegada de Xi Jinping (2012) a la presidencia del país se ha propuesto el avance de China como líder global (Yang, 2021).

El gobierno chino asumió estrategias alternativas para continuar expandiéndose, considerando que el crecimiento económico a largo plazo puede lograrse no solo a través de la inversión de capital, sino también a través de tecnología e innovación. Por lo tanto, el liderazgo chino en el desarrollo de la nueva red de telecomunicaciones no es accidental, el desarrollo 
tecnológico se ha convertido en el eje de la visión estratégica china bajo la presidencia de Xi Jinping: uno de los principales objetivos de su agenda política es convertir a China de ser la "fábrica del mundo" en un centro de innovación (Mariani y Bertolini, 2019).

Bajo este enfoque de largo plazo, China espera que la ciencia y la tecnología contribuyan al $60 \%$ de su PIB, y que el 2,5\% del mismo se destine a su investigación y desarrollo, lo cual la llevaría a ser una de las naciones con el más alto índice de participación en este sector. Para el logro de estos objetivos, China se propuso construir cuarenta y cinco Centros de Innovación para 2025 y en 2020 tener un promedio de 100 robots por cada 100 mil trabajadores. De igual modo, prevé arribar al año 2025 con un 84 \% de automatización por control numérico en sus principales procesos de manufactura y un $64 \%$ en digitalización en herramientas de diseño. Lo anterior, procurando el desarrollo de las capacidades de innovación nacional y promoviendo la formación de talentos (Citado en Oropeza, 2019: 188).

La tecnología de punta (high-tech) refiere a la tecnología en su mayor etapa de desarrollo y la más avanzada que se encuentra disponible. China, a raíz de su enfoque en la innovación y el desarrollo tecnológico, ha buscado su posicionamiento en los sectores high-tech, como son la inteligencia artificial, la robótica, los drones y la realidad virtual, entre otros.

La IA es la superpotencia de esta era y la principal respuesta a la búsqueda de China por dominar el mundo; se afirma que para 2030 el país quiere convertirse en el centro de innovación de IA más grande del mundo, y se prevé que la industria de la IA alcance una valoración de 202,57 mil millones de dólares para 2026 (GBO, 2020).

Considerando que la IA se ha convertido en un nuevo motor de desarrollo económico y una oportunidad de desarrollo social, el Plan de Desarrollo de Inteligencia Artificial de Nueva Generación de 2017 clarificó la estrategia china y planteó sus tres objetivos estratégicos en el mediano plazo:

1. Para 2020, la tecnología y las aplicaciones de IA alcanzarán un nivel globalmente avanzado, la industria de la IA se convierte en un nuevo punto de crecimiento económico y su aplicación será en un nuevo enfoque hacia mejorar los medios de vida de las personas para respaldar el objetivo chino de convertirse en un país impulsado por la innovación y construir una sociedad próspera.

2. Para el año 2025, la tecnología y la aplicación de IA alcanzan un nivel globalmente avanzado, la IA se convierte en una fuerza impulsora importante para la actualización industrial y la reestructuración económica.

3. En 2030 la teoría, la tecnología y las aplicaciones de la IA alcanzan un nivel globalmente avanzado. China se convierte en un centro global de innovación en IA, mientras que la economía y la sociedad de la inteligencia progresan notablemente, sentando una base sólida para convertirse en un país impulsado por la innovación y económicamente poderoso (Department of International Cooperation Ministry of Science and Technology [MOST], 2017).

En general, el avance tecnológico, utilizado en conjunto se puede emplear para mejorar las características y desempeño de cada producto, en el caso de la IA se puede emplear para resolver problemas, es posible utilizarla ampliamente en el diseño, la configuración y optimización para las redes 5G (Xiaohu, et al., 2018). Por ende, la 5G y la inteligencia artificial van de la mano y su utilización conjunta puede optimizarlas. Incluso se afirma que la $5 \mathrm{G}$ y el desarrollo de IA determinará la superioridad económica y bélica en los próximos años (Fricke, 2020).

Otro sector muy importante en el que China se beneficia a raíz del avance de la tecnología y de la 5G, es la industria manufacturera. Se está adoptando cada vez más en la producción y logística, al grado en que esta se convertirá en el motor que impulsa la transformación digital de todas las industrias y el desarrollo de la economía digital (Xiang, 2020). 
La 5G, de acuerdo con sus características, posibilita la conexión de más dispositivos, garantiza que las conexiones sean ultra fiables y seguras para evitar la pérdida de datos, así como la captura en tiempo real. Estas particularidades posibilitan que distintas aplicaciones utilicen tales beneficios en diferentes áreas, como el mantenimiento predictivo avanzado, el monitoreo y control de precisión para maximizar la productividad y reducir las tasas de defectos, así como la realidad aumentada y experto remoto para mejorar la eficiencia (STL Partners, 2020).

De esta manera se mejora la eficiencia y productividad, reduciendo costos y tiempos de inactividad; además, hay un seguimiento del correcto funcionamiento de las cosas, sin necesidad de esperar a que fallen, y se puede tener ayuda y soporte externo en tiempo real haciendo una visita virtual.

Los fabricantes ya no necesitarán depender de redes fijas para sus necesidades de comunicación, las fábricas en China cada vez tienen una mejor tecnología y se encuentran mejor conectadas; la integración de la 5G, considerando las ventajas mencionadas, hará posible un mayor y mejor monitoreo de los procesos de producción, más eficiencia, velocidades más rápidas de producción y una mayor precisión del equipo utilizado.

Otro instrumento en los sectores de punta son los drones, se han empleado en el combate a la covid-19, en el sector agrícola, en el ramo turístico, como apoyo para combatir desastres naturales e, incluso, como una herramienta de cooperación técnica entre países. China se ha convertido en un productor líder de plataformas de vehículos aéreos no tripulados (UAV, por sus siglas en inglés) para uso comercial y militar, y sus tecnologías se están utilizando de formas sin precedentes (Oliver, 2020).

Además de los drones, en general China ha tenido un avance significativo en el sector de la robótica, según el informe del Instituto Chino de Electrónica, este país cuenta con el mercado de robots industriales más grande del mundo, con un valor estimado de 5.700 millones de dólares en 2019, el gobierno también ha promovido un plan de desarrollo para la industria de la robótica de 2016 a 2020 con el objetivo de impulsar la investigación y el desarrollo de tecnologías robóticas clave, y el fomento de importantes innovaciones, así como la promoción de la cooperación internacional en el campo (Xinhua, 2019).

El panorama en la robótica ha cambiado en los últimos años, varias empresas extranjeras han sido adquiridas por grupos chinos lo que ha fortalecido su posicionamiento en el área, China es el primer país del mundo en la aplicación de robots industriales y está aumentando y diversificando cada vez más su uso. Los países líderes en la aplicación de robots industriales, además de China, son Corea, Japón, Estados Unidos y Alemania (Karabegović, 2016).

Debido al avance en la tecnología y la producción de robots, su uso en la industria ha ido en aumento, las principales industrias que han adoptado robots en este país son las automotrices (44,5\%), electrónica $(24,7 \%)$, metales (13,9\%), plásticos y productos químicos (11,5\%), así como alimentos y bebidas (2,9\%) (Cheng, et al., 2019). Además de su uso en la industria, se está difundiendo la utilización de los robots en sectores como la salud, la manufactura, la seguridad e incluso se aprecia su utilización para fines de esparcimiento y entretenimiento. En general, la 5G está teniendo un impacto directo en la evolución y difusión del internet de las cosas, el mejoramiento de la conectividad y el desarrollo de sectores estratégicos en los que China está adquiriendo un papel transcendental y un liderazgo internacional. 


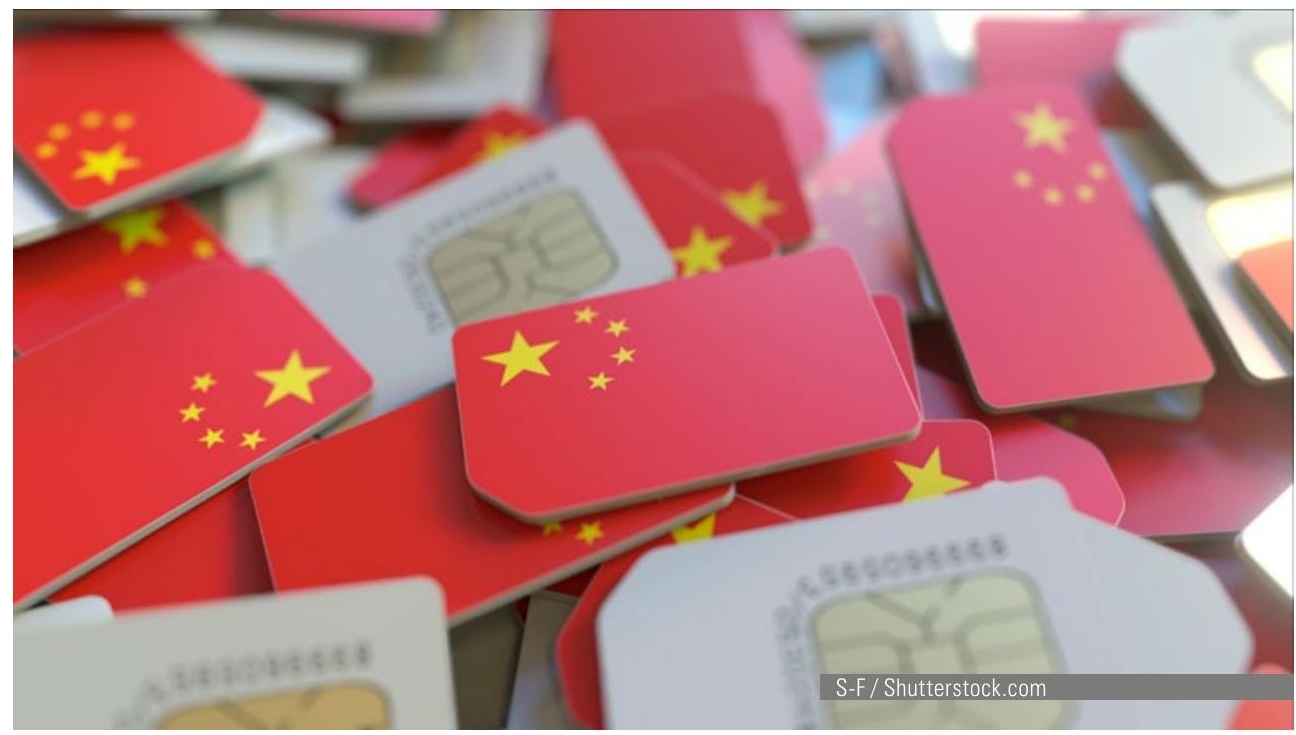

\section{Conclusiones}

Los avances tecnológicos en la historia han modificado los sectores productivos y por ende las relaciones económicas, cada uno de estos avances tiene dimensiones que no pueden catalogarse en un área única y determina$\mathrm{da}$, sino que tienen efectos directos en diversos sectores. Definitivamente la tecnología 5G es uno de los mayores avances tecnológicos de la actualidad, su uso llegó para transformar las relaciones económicas, sociales y políticas en la economía global.

La $5 G$ está revolucionando el mundo debido a sus altas velocidades de descarga, la excelente eficiencia energética, la baja latencia y su gran capacidad de soporte; considerando esta evolución, la llamada cuarta revolución industrial está en camino y la vida diaria está modificándose en todas las esferas, desde la económica, la educación, la salud, China tiene el primer lugar en el mundo al liderar este avance tecnológico.

Muchos sectores tecnológicos están expandiendose en China y el avance de la $5 \mathrm{G}$ ha tenido una influencia directa en esta expansión, particularmente en sectores clave que no solo impactan en la vida diaria de la sociedad, sino que permitirán el fortalecimiento de sectores fundamentales para el posicionamiento del país en el plano internacional.

Estados Unidos y China son los dos principales países que se disputan la supremacía tecnológica 5G. El impulso y la evolución de la 5G en China ha impactado en el desarrollo de sectores estratégicos para su nación, generando ventajas económicas, sobresaliendo en el mundo y, a su vez, como consecuencia está dominando la carrera tecnológica con Estados Unidos. De acuerdo con el artículo la hipótesis planteada tiene sentido porque el desarrollo y avance de la tecnología 5G le está permitiendo ganar presencia con este tipo de tecnología, adquiriendo mayor supremacía económica en comparación con Estados Unidos.

De hecho, es posible afirmar que la extensión de la guerra comercial de 2018-2021 entre ambos países en el frente tecnológico es la manifestación más clara acerca de su importancia. Por lo desarrollado en este artículo, se vislumbra que la balanza se inclinará hacia China, lo que, sin duda, marcaría un hito en la disputa por la hegemonía económica global que ha mantenido Estados Unidos hasta la actualidad. 
El frente tecnológico se equilibra con tendencia a que China adquiera un predominio mayor en este ámbito, dando surgimiento de un NOEl sinocéntrico que enfrentaría y remplazaría a las instituciones creadas en la era del dominio estadounidense, en el contexto de un sistema económico internacional, que seguramente estará fragmentado. Independientemente, pero no al margen de la disputa hegemónica internacional, se desea que la 5G constituya un puente de beneficios para la sociedad en su escala local y global dinamizando la economía, la sociedad, la política y el medio ambiente, entre otros.

\section{Referencias}

- Banco Mundial. (2021). Base de datos de crecimiento. https://datos.bancomundial.org/indicator/NY.GDP. MKTP.KD.ZG?locations $=\mathrm{CN}$

- Batista, M y Díaz, E. (2019). Tecnología móvil 5G. Mare Ingenii. Ingenierías 1(1). http://cipres.sanmateo. edu.co/index.php/mi

- $\quad$ BBC. (01 de diciembre de 2020). China-US Trade War: Beijing Escalates tit-for-tat with Washington. https://www.bbc.com/news/business-55132425

- CAICT. (2017). $5 \mathrm{G}$ in China: Outlook and Regional Comparisons. https://data.gsmaintelligence.com/ api-web/v2/research-file-download?id=28999734\&file =5G\%20in\%20China\%200utlook\%20and\%20 regional\%20comparisons.pdf

- $\quad$ Cheng, H. Ruixue, J., Dandan L., y Hongbin L. (2019). The Rise of Robots in China. Journal of Economic Perspectives, 33 (2): 71-88.

- Cornejo, R. y González, G. (2009). Ciencia y tecnología en China. Comercio Exterior, Vol. 59. No. 9. Pp. 724-734.

- Corral, D. (2020). 5G, una carrera por la hegemonía y el futuro con muchos beneficios. Documento Marco IEEE 07/2020. http://www.iee.es/Galerias/fichero/docs_marco/2020/DIEEEM06_2020DAVCOR_5G.pdf y/o enlace bie3

- Crammer-Flood, E. (2021). Global Ecommerce Update 2021. E Marketer. https://www.emarketer.com/ content/global-ecommerce-update-2021

- Department of International Cooperation Ministry of Science and Technology (MOST). (2017). Next Generation Artificial Intelligence Development Plan Issued by State Council. China science and technology. No. 17.

- GBO. (02 de julio de 2020). Will China Become Leader in Al by 2030? https://www.globalbusinessoutlook. com/china-ai/

- García, A., Iglesias, E., Kim, K. y Park, S. (2020). 5G The Driver for the Next-Generation Digital Society in Latin America and the Caribbean. Inter-American Development Bank. Connectivity, Markets and Finance Division. VI. Series.

- González, J. (2020). El comercio electrónico en México y China. Surgimiento, evolución y perspectivas. México y la Cuenca del Pacífico. Vol. 9 No. 27 Pp. 53-84 http://www.mexicoylacuencadelpacifico.cucsh. udg.mx/index.php/mc/article/view/688D0l: 10.32870/mycp.v9i27.688

- González, J. y Salamanca, 0. (2016). El camino hacia la tecnología 5G. Télématique, 15(1),27-47. https:// www.redalyc.org/articulo.oa?id $=784 / 78445977002$

- Global Times (29 de julio de 2021). Living in China's Technological Miracle CPC's innovation-driven strategy powers xiaokang society. https://www.globaltimes.cn/page/202107/1230033.shtml

- Donahue, T. (2019). The Worst Possible Day: U.S. Telecommunications and Huawei. PRISM, 8(3), 14-35. doi:10.2307/26864274

- DTTL. (2020). Combating Covid-19 with 5G Opportunities to Improve Public Health systems. Recuperado de: https://itreseller.com.pl/wp-content/uploads/2020/03/Huawei_Deloitte_Raport_Zwalczanie-covid-19przy-pomocy-5G_Możliwości-usprawnienia-systemów-publicznej-öpieki-zdrowotnej.pdf

- Fricke, B. (2020). Artificial Intelligence, $5 G$ and the Future Balance of Power. Konrad Adenauer Stiftung. doi:10.2307/resrep25281

- Kahuha. E. (17 de enero, 2021). 5 Real Life Use Cases of $5 G$ Ultra-Reliable Low-Latency Communication (URLLC). Section. https://www.section.io/engineering-education/five-real-life-use-cases-of-5g-ultrareliable-low-latency-communication-urllc/

- Kania, E. (2019). Securing Our 5G Future: The Competitive Challenge and Considerations for U.S. Policy (pp. 6-7, Rep.). Center for a New American Security. doi:10.2307/resrep20451.4

- Karabegović, I. (2016). The Role of Industrial Robots in the Development of Automotive Industry in China. International Journal of Engineering Works, 3(12), 92-97.

- Kennedy, S. (2020). Washington's China Policy Has Lost Its Wei. Center for Strategic and International Studies (CSIS). doi:10.2307/resrep25232

- $\quad$ Kshetri, N. (2018). 5G in E-Commerce Activities. IT Professional. 20. 73-77. 10.1109/MITP.2018.043141672. 
- Kwak, J., Zhang, Y., y Yu, J. (2019). Legitimacy Building and E-Commerce Platform development in China: The experience of Alibaba. Technological Forecasting and Social Change, 139, 115-124.

- Lu, X., y Sun, Y. (2020). Application of 5G Technology in Education Informatization. Strategic Study of Chinese Academy of Engineering, $21(6), 120-128$

- Mariani, L., y Bertolini, M. (2019). The US-China 5G Contest: Options for Europe. Istituto Affari Internazionali (IAI). doi:10.2307/resrep19676

- Ministry ofCommerce. (2020).E-commerce inChina 2019.http://www.gov.cn/xinwen/202007/02/5523479/ files/0a2c57d8ba6d4e26b83d96cdd764d6f0.pdf

- Mokhtar, Umaimah \& Bin Ahmad, Jaidi. (2020). 5G Communications : Potential Impact on Education Technology In Higher Ed.

- Oliver, R. (2020). The Strategic Implications of Chinese UAVs: Insights from the Libyan Conflict. China Brief Vol. 20, No. 15.

- Oropeza, A. (2019). China. La construcción del poder en el siglo XXI. México: Universidad Nacional Autónoma de México/ Instituto de Investigaciones Jurídicas.

- Ouyang, R. (9 de julio de 2020). 5G's Indispensable Role in China's Fight Against Covid-19. CGTN. https://news.cgtn.com/news/2020-07-09/5G-s-indispensable-role-in-China-s-fight-against-covid-19RXRu9TIZ9S/index.html

- Patel, S., Shah, V. y Kansara, M. (2018). Comparative Study of 2G, 3G and 4G. International Journal of Scientific Research in Computer Science, Engineering and Information Technology, 3(3), 1962-1964. https://www.researchgate.net/publication/327764127_Comparative_Study_of_2G_3G_and_4G

- PWC. (2021). The Global Economic Impact of 5G. https://www.pwc.com/gx/en/tmt $/ 5 \mathrm{~g} / \mathrm{global}-\mathrm{economic}$ impact-5g.pdf

- Rogers, M. y Nye, G. (12 de octubre de 2019). Why America Must Boldly Win the Technological Race Against China. The Hill. https://thehill.com/opinion/technology/466705-why-america-must-boldly-win-thetechnological-race-against-china

- Rühlig, T. y Björk, M. (2020). What to Make of the Huawei Debate? 5G Network Security

- $\quad$ and Technology Dependency in Europe.

- Rühlig, T., Seaman, J., y Voelsen, D. (2019). 5G and the US-China Tech Rivalry-a Test for Europe's Future in the Digital Age. German Institute for International and Security Affairs (SWP Comment 29), Berlin. https://www. ifri. org/sites/default/files/atoms/files/us-china_5g_and_europe__swp_comment_june_2019. pdf.

- Soldani, D. (2020). Fighting Pandemics by Exploiting 5G, Al and Bigdata Enabled Technologies: How 5G Can Help Contain the Spread of Covid-19. Journal of Telecommunications and the Digital Economy, 8(2), 146.

- Statista. (2021). Number of internet users in China from 2008 to 2020. https://www.statista.com/ statistics/265140/number-of-internet-users-in-china/

- State Council. (2020). E-commerce a Driver for China's Economic Development. http://english.www.gov. cn/statecouncil/ministries/202007/03/content_WS5efe8c8fc6d05a0f89706982.html

- STL Partners. (2020). 5G's Impact on Manufacturing: \$740bn of benefits in 2030. Executive Briefing. https:// carrier.huawei.com/ /media/CNBGV2/download/program/Industries-5G/5G-Impact-on-Manufactureing.pdf

- Tamayo, R. (2017). 5G: Una nueva generación para una nueva sociedad. HAMUT'AY, 1(1), 28-32.

- Tekir, G. (2020). Huawei, 5 G Networks, and Digital Geopolitics. International Journal of Politics and Security (IJPS), Vol. 2, No. 4, pp.113-135.

- Tomás, J. (10 de septiembre de 2020). Beijing Becomes Second Chinese City to Reach Full $5 \mathrm{G}$ Coverage. RCR WIRELESS NEWS. https://www.rcrwireless.com/20200910/5g/beijing-becomes-second-chinesecity-reach-full-5g-coverage

- UNFPA. (2021). Población Mundial China. https://www.unfpa.org/es/data/world-population/CN

- West, D. M. (2016). How 5G Technology Enables the Health Internet of Things. Brookings Center for Technology Innovation, 3, 1-20.

- Wong, D. y Chipman, A. (2020). The US-China Trade War: A Timeline. China Briefing. https://www.chinabriefing.com/news/the-us-china-trade-war-a-timeline/

- Xiang, L. (2020). How 5G Will Revolutionize Manufacturing 5G is poised to take productivity to the next level. Huawei. https://www.huawei.com/en/technology-insights/publications/winwin/38/5g-revolutionizemanufacturing

- Xiaohu, Y., Zhang, C., Tan, X., Jin, S. y Wu, H. (2018). Al for 5G: Research Directions and Paradigms. Science China. Information Sciences.

- Xinhua. (25 de Agosto de 2019). China's robotics industry forges ahead to brighter future. http://www. xinhuanet.com/english/2019-08/25/c_138337432.htm

- Yang, Y. (2021). China's Strategic Narratives in Global Governance Reform under Xi Jinning, Journal of Contemporary China, 30:128, 299-313. https://doi.org/10.1080/10670564.2020.1790904

- Zhen, L. (12 de diciembre de 2020). US-China Trade War: Joe Biden Hints he Will Keep Up Pressure on Beijing with Pledge to Fight 'Unfair Trade Practices'. South China Morning Post. https://www.scmp.com/ news/china/diplomacy/article/3113726/us-china-trade-war-joe-biden-hints-he-will-keep-pressure 